\title{
Differential Effect of MyD88 Signal in Donor T Cells on Graft-versus-Leukemia Effect and Graft-versus- Host Disease after Experimental Allogeneic Stem Cell Transplantation
}

\author{
Ji-Young Lim', Da-Bin Ryu', Sung-Eun Lee', Gyeongsin Park'*, Eun Young Choi , and Chang-Ki Min ${ }^{1, *}$
}

\begin{abstract}
Despite the presence of toll like receptor (TLR) expression in conventional TCR $\alpha \beta T$ cells, the direct role of TLR signaling via myeloid differentiation factor 88 (MyD88) within $T$ lymphocytes on graft-versus-host disease (GVHD) and graft-versus-leukemia (GVL) effect after allogeneic stem cell transplantation (allo-SCT) remains unknown. In the allo-SCT model of C57BL/6 $\left(H-2^{b}\right) \rightarrow B 6 D 2 F 1\left(H-2^{b / d}\right)$, recipients received transplants of wild type (WT) T-celldepleted (TCD) bone marrow (BM) and splenic $T$ cells from either WT or MyD88 deficient (MyD88KO) donors. Hosttype (H-2 $\left.{ }^{d}\right)$ P815 mastocytoma or L1210 leukemia cells were injected either subcutaneously or intravenously to generate a GVHD/GVL model. Allogeneic recipients of MyD88KO T cells demonstrated a greater tumor growth without attenuation of GVHD severity. Moreover, GVHDinduced GVL effect, caused by increasing the conditioning intensity was also not observed in the recipients of MyD88KO T cells. In vitro, the absence of MyD88 in T cells resulted in defective cytolytic activity to tumor targets with reduced ability to produce IFN- $\gamma$ or granzyme $B$, which are known to critical for the GVL effect. However, donor $T$ cell expansion with effector and memory T-cell differentiation were more enhanced in GVHD hosts of MyD88KO T cells. Recipients of MyD88KO T cells experienced greater expansion of Foxp3- and IL4-expressing T cells with reduced INF-y producing $T$ cells in the spleen and tumor-draining lymph nodes early after transplantation. Taken together, these results highlight a differential role for MyD88 deficiency on donor T-cells, with decreased GVL effect without attenuation of the GVHD severity after experimental allo-SCT.
\end{abstract}

\footnotetext{
${ }^{1}$ Department of Internal Medicine, ${ }^{2}$ Department of Pathology, Seoul St. Mary's Hospital, College of Medicine, The Catholic University of Korea, Seoul 137-701, Korea, ${ }^{3}$ Department of Biomedical Sciences, Seoul National University College of Medicine, Seoul 110-799, Korea

*Correspondence: ckmin@catholic.ac.kr (CKM); gspark@catholic.ac.kr (GP)
}

Received 2 June, 2015; revised 28 July, 2015; accepted 30 July, 2015; published online 10 November, 2015

Keywords: GVHD, GVL effect, MyD88, T cell, Treg and allo-SCT

\section{INTRODUCTION}

Graft-versus-host disease (GVHD) is a major complication following allogeneic stem cell transplantation (allo-SCT) and a substantial determinant of non-relapse morbidity and mortality. GVHD is a complex process involving dysregulated inflammatory cytokine cascades and distorted responses of donor cellular effectors to host alloantigens (Hill and Ferrara, 2000). Alloreactive donor T cells are important effector cells in the development of GVHD, and proinflammatory cytokines enhance the generation of donor anti-host cytotoxic function (van den Brink and Burakoff, 2002). Concurrently, the graft-versus-leukemia (GVL) effect provided by an allograft represents a very potent form of immune therapy against malignancy (Appelbaum, 2001). Unfortunately, GVHD and GVL effect are tightly linked as demonstrated by the inverse correlation between leukemia relapse rates and the severity of GVHD (Horowitz et al., 1990). Prevention of GVHD by T-cell depletion or nonspecific immune suppression is associated with increased risk for leukemia relapse after allo-SCT (Barrett, 1997).

Toll-like receptors (TLRs) recognize pathogen-associated molecular patterns that are present on microorganisms and also recognize endogenous ligands released from damaged tissue (Wu et al., 2007). The interaction of the commensal microbiota with TLRs plays an important role in the innate immune response whose recognition can regulate the adaptive immune response through multiple mechanisms. All TLRs, except TLR3, can signal through an adaptor molecule known as myeloid differentiation primary response gene (88) (MyD88), which leads to molecular translocation of NF-kB, with consequent upregulation of proinflammatory cytokines and chemokines. The MyD88 signaling adaptor operates in many cell types downstream of TLRs and IL-1 receptor family members. However, cell-type-specific functions of MyD88 signaling remain poorly characterized. TLRs can trigger activation of several arms of the adaptive immune response, generating effectors including immunoglobulin, $\mathrm{TH} 1$, and TH17 CD4 effector T cells, and CD8 effector T cells. The MyD88 pathway is known to be important in generating $\mathrm{TH} 1$ responses (Schnare et al., 2001) and activation of MyD88 has also been shown to be required for colitogenic CD4 T cells in murine models of inflammatory bowel disease (Fukata et al., 2008; Tomita et al., 2008). 
Emerging studies indicate that activating TLR-MyD88 signals within $\mathrm{CD} 4^{+}$or $\mathrm{CD}^{+} \mathrm{T}$ cells can enhance its antitumor activity. In CD4 T-helper cells, TLR1/2, TLR5, TLR7/8, and TLR9 engagement has been shown to enhance IL-2 production (Bendigs et al., 1999; Caron et al., 2005; KomaiKoma et al., 2004). TLR2 or TLR9 ligation on CD4 ${ }^{+}$and $\mathrm{CD}^{+} \mathrm{T}$ cells also enhances survival by modulating the expression levels of antiapoptotic proteins, including A1, bcl2, and bcl-xl (Asprodites et al., 2008; Cottalorda et al., 2006; Gelman et al., 2004; Zheng et al., 2008). TLR1/2 stimulation on $\mathrm{CD}^{+} \mathrm{T}$ cells has also been shown to enhance IFN- $\gamma$ production and increase cytotoxicity in vitro (Asprodites et al., 2008; Cottalorda et al., 2006). Amplifying TLR-MyD88 signals within tumor-specific $\mathrm{T}$ cells enhanced antitumor activity to suboptimal levels of weakly immunogenic tumor antigens (Hartman et al., 2010). Ligand-independent TLR signals generated by ectopic overexpression of MyD88 has been shown to provide local and systemic antitumor immunity (Hartman et al., 2010).

Although several studies have demonstrated important roles of MyD88 in T cells, little is known about their potential function in GVHD and/or GVL effect. Furthermore, how donor-type Tcell differentiation could be regulated by MyD88 in the setting of allo-SCT remains unclear. Herein, we demonstrate that the absence of MyD88 in donor T cell diminishes the GVL effect without attenuating the acute GVHD (aGVHD) severity following experimental allo-SCT. Alloreactive effector/memory T-cell differentiation was more greatly enhanced in the aGVHD hosts with MyD88-deficient T cells, but in the GVL setting, MyD88 deficiency in donor $T$ cells contributed to regulatory $T$ cell (Treg) and $\mathrm{TH} 2$ differentiation, but not to $\mathrm{TH} 1$ differentiation. Thus, our findings reveal a novel mechanism for dissociation between the aGVHD and GVL effect according to the innate adaptor MyD88 of donor $\mathrm{T}$ cell.

\section{MATERIALS AND METHODS}

\section{Mice}

Female C57BL/6 (B6, H-2 ${ }^{\text {b }}$, B6.Ly-5a (CD45. $\left.1^{+}\right)$, and B6D2F1 $\left(\mathrm{F} 1, \mathrm{H}-2^{\mathrm{b} / \mathrm{d}}\right)$ mice (8- to 12-week old) were purchased from Japan SLC Inc. (Japan). MyD88 deficient (MyD88KO, H-2 ${ }^{b}$ ) mice were generated by Kawai et al. (1999) and had been backcrossed $>10$ generations onto the C57BL/6J strain.

\section{Experimental allo-SCT and tumor cell inoculation}

Mice underwent transplantation using a standard protocol described previously (Lim et al., 2011; Min et al., 2004). Briefly, B6D2F1 (F1) recipients received T-cell depleted bone marrow (TCD BM) cells $\left(5 \times 10^{6}\right)$ plus $1 \times 10^{6}$ purified T cells from allogeneic C57BL/6 (B6) mice after total body irradiation (TBI) with $900,1,100$ or 1,300 cGy. B6.Ly-5a (CD45. $\left.1^{+}\right)$mice were used to identify donor $\mathrm{T}$ cells in various organs. The degree of systemic GVHD was assessed using a scoring system that incorporates five clinical parameters: weight loss, posture (hunching), activity, fur texture and skin integrity (Cooke et al., 1998). A subcutaneous (s.c.) injection of $1 \times 10^{6}$ cells of P815 $\left(\mathrm{H}-2^{\mathrm{d}}\right)$ mastocytoma or L1210 cells into a flank of F1 mice on day 1 induced locally invasive, fast growing tumors. Tumor size measurements were made every 3 or 4 days after s.c. tumor inoculation by measuring largest orthogonal diameters with a caliper, and were recorded as tumor volumes $\left(\mathrm{mm}^{3}\right)$. Some mice concurrently received $3 \times 10^{3}$ cells of P815 intravenously (i.v.) and leukemic death was defined by death with occurrence of either macroscopic tumor nodules in liver or spleen or hind leg paralysis.
Histological analysis and immunohistochemistry Formalin-fixed, paraffin-embedded tissue sections were stained with $\mathrm{H} \& \mathrm{E}$, and examined by a pathologist using a semiquantitative scoring system for abnormalities known to be associated with GVHD. The scoring system for each parameter that evaluated both the extent and severity of tissue damage denoted 0 as normal, 0.5 as focal and rare, 1 as focal and mild, 2 as diffuse and mild, 3 as diffuse and moderate, and 4 as diffuse and severe (Cooke et al., 1998). For TNF- $\alpha$ staining, sections were rehydrated, endogenous peroxidase was eliminated, incubated with TNF- $\alpha$ (Santa Cruz, USA) antibody followed by HRP conjugated secondary antibody and developed with DAB system.

\section{Flow cytometric analysis}

Single-cell suspensions were stained in FACS buffer at $4^{\circ} \mathrm{C}$ for 30 min. Intracellular cytokine to detect Th subsets was measured as previously described (Lim et al., 2014). Samples were analyzed using an LSRII (BD Pharmingen; USA). The following antibodies against mouse antigens were purchased from $B D$ Pharmingen (USA): FITC-conjugated anti-H-2d, anti-IL-17A, and anti-IL-4; and PE-conjugated anti-CD8; and PerCP-Cy5.5conjugated anti-CD62L and anti-CD8; and APC-conjugated anti-CD44, anti-IFN $\gamma$, anti-Foxp3, Annexin V; and APC-Cy7conjugated anti-CD4. Alexa Fluor 700-conjugated anti-CD45.1 was purchased from ebioscience (USA).

\section{In vivo proliferation of donor T cells}

Purified donor $\mathrm{T}$ cells were labeled with $2 \mu \mathrm{M}$ carboxyfluorescein diacetate succinimidyl ester (CFSE; Molecular Probes, Inc.) for $10 \mathrm{~min}$ at $37^{\circ} \mathrm{C}$. These CFSE labeled cells were then resuspended and infused into recipient mice. Splenocytes from recipient mice were harvested 4 days after transplantation, stained with APC-Cy7-conjugated anti-CD4 and PerCPCy5.5-congugated anti-CD8, washed with $1 \times$ PBS and assessed for FACS analysis.

\section{Cytometric bead analysis}

The concentrations of six cytokines (IFN- $\gamma$, IL-6, TNF- $\alpha$, MCP-1, RANTES and IL-17A and IL-10) in recipient sera or culture supernatants were determined using a commercially available kit (BD Pharmingen). All tests were performed according to the manufacturer's instructions.

\section{ELISA}

The concentrations of granzyme $B$ in culture supernatants were determined using a kit (R\&D Systems, USA) according to the manufacturer's protocol.

\section{$R T-P C R$}

To detect T-bet, GATA3, Foxp3, and ROR $\gamma T$ mRNA expression, real-time quantitative PCR (qPCR) was performed using a SYBR Green Master Mix and run in a CFX96 real-time thermal cycler (Bio-Rad, USA). The following primers were used: murine T-bet primers: forward, 5'-CCCACAAGCCATTACAGGAT G-3', and reverse, 5'-TATAAGCGGTTCCCTGGCATG-3'; murine GATA3 primers: forward, 5'-AGGAGTCTCCAAGTGTGCG AA-3', and reverse, 5'- TTGGAATGCAGACACCACCT-3'; and murine Foxp3 primers: forward, 5'-ACAACCTGAGCCTGCAC AAGTT-3', and reverse, 5'-GCCCACCTTTTCTTGGTTTTG-3'; and murine $R O R \gamma T$ primers: forward, 5'-TGGAAGATGTGGAC TTCGTTT-3', and reverse, 5'- TGGTTCCCCAAGTTCAGGAT3'; and murine GAPDH primers: forward, 5'-GGTGTGAACGGA TTGCCGTATT-3', and reverse, 5'-GGCCTTGACTGTGCCGTT AATTT-3'. 


\section{Cytotoxicity assays}

Standard allogeneic mixed lymphocyte reaction (MLR) was performed using naïve C57BL/6 splenic CD3 ${ }^{+} \mathrm{T}$ cells $\left(2 \times 10^{5}\right)$ as responders and irradiated naïve BDF1 T-cell depleted mononuclear cells $\left(2 \times 10^{5}\right)$ as stimulators. After 4 days, CD8 ${ }^{+}$ effector cells were purified and cultured with target P815 or EL4 cells for $4 \mathrm{~h}$. Cytotoxicity assay was conducted using nonradioactive lactate dehydrogenase release using a cytotoxicity detection kit (CytoTox 96, Promega, USA) according to the manufacturer's instructions. Spontaneous release and maximum release were determined by incubating target cells without effector cells in medium alone or in $0.5 \%$ NP40, respectively. The percent cytotoxicity was calculated as follows: (experimental release - spontaneous release) / (maximum release - spontaneous release) $\times 100 \%$.

\section{Statistical procedures}

All values are expressed as means \pm standard errors (SEMs). Comparisons between groups were performed using the parametric independent samples $t$-test if the groups contained more than five animals, or using the Mann-Whitney test otherwise. The Wilcoxon rank-sum test was used to analyze survival data.

\section{RESULTS}

Deficiency of MyD88 in donor T cells mitigates GVL effect To examine the contribution of the presence of MyD88 signaling in donor T cells in regulating GVL effect and aGVHD development, we used a B6 $\left(\mathrm{H}-2^{\mathrm{b}}\right) \rightarrow \mathrm{F} 1\left(\mathrm{H}-2^{\mathrm{b} / \mathrm{d}}\right)$ experimental alloSCT model, which differs at major and minor histocompatibility loci. Lethally irradiated recipient mice were transplanted with wild-type $(\mathrm{WT})$ TCD-BM $\left(5 \times 10^{6}\right)$ together with either WT or MyD88KO mice spleen T cells $\left(1 \times 10^{6}\right)$ on day 0 . Thereafter, host-type $\left(\mathrm{H}-2^{\mathrm{d}}\right) \mathrm{P} 815$ mastocytoma or L1210 leukemia cells (1 $\times 10^{6}$ ) were injected subcutaneously on day 1 , meaning that only the transferred donor $\mathrm{T}$ cells lack the MyD88-dependent pathway within the recipient mice. As shown in Fig. $1 \mathrm{~A}$, subcutaneous tumors in the allogeneic recipients receiving MyD88KO $\mathrm{T}$ cells exhibited markedly increased growth in vivo compared to those receiving WT T cells (tumor volume on day 42, 15205.6 vs. $373.9 \mathrm{~mm}^{3}, \mathrm{P}<.001, \mathrm{P} 815$ and 8311.5 vs. 3200.7 $\mathrm{mm}^{3}, \mathrm{P}<.01, \mathrm{~L} 1210$, respectively). Next, P815 cells were injected intravenously $\left(3 \times 10^{3}\right)$. At 70 days post-allo-SCT, as shown in Fig. 1B, $50 \%$ of allogeneic recipients of WT T cells died due to severe aGVHD, as necropsy showed no evidence of tumor. In contrast, $83.5 \%$ of those of MyD88 KO T cells died with clinical and microscopic evidence of tumors $(P<.05)$. Consistently, the weights of the spleen and liver were significantly increased in the latter as compared with the former. We further evaluated the GVL effect mediated by increasing intensity (1,300 vs. $900 \mathrm{cGy}$ ) of TBI conditioning (Lim et al., 2011) according to the presence of MyD88 signaling. Enhanced GVL effect in the allogeneic recipients receiving 1300 cGy TBI was abolished in the recipients of MyD88KO T cells, suggesting that the absence of MyD88 in donor T cells led to a lack of aGVHDinduced GVL effect caused by a higher TBI dose (Fig. 1C).

In vitro, the absence of MyD88 in T cells resulted in defective $T$ cell functions, such as cytolytic activity to tumor and the ability to secrete pro-inflammatory cytokines to host antigens. We examined the effects of MyD88 signaling in donor T cells on cytolytic activity to host antigens. Splenocytes harvested from MyD88 KO mice showed significantly reduced cytolytic activity against host type P815 targets compared to those from WT mice (Fig. 1D). In addition, MyD88KO or WT B6 T cells were activated by anti-CD3/anti-CD28 antibodies and allogeneic mixed leukocyte reaction using F1 stimulator cells. T cells from MyD88 KO mice showed a significant reduction in the production of proinflammatory cytokines and effector molecules including IFN- $\gamma$, IL-6, granzyme B, and IL-17A with significant augmentation in IL-10 as compared with WT T cells (Fig. 1E). Taken together, our data demonstrate a critical role of MyD88 signaling in donor T cells for the maintenance of the GVL effect in the allo-SCT setting.

\section{Absence of MyD88 signal in donor T cells does not attenuate the GVHD severity}

After allo-SCT, GVL effect is associated with GVHD severity as demonstrated by the inverse correlation between leukemia relapse rates and the severity of GVHD (Horowitz et al., 1990). In this study, however, clinical aGVHD scores and GVHD survival were comparable between the allogeneic recipients of WT T cells and MyD88KO T cells with TCD-BM (Fig. 2A). Furthermore, we observed that the severity of aGVHD assessed by histological grade was not significantly different in liver, lung, thymus, small intestine, and colon of recipients that received MyD88KO donor T cells compared with those that received WT donor $\mathrm{T}$ cells (Fig. 2B). To directly evaluate the target tissue injury, we examined the expressions of TNF- $\alpha$, the primary cytokine associated with aGVHD-induced damage in each organ, and observed similar expression between the two groups (Fig. 2C). In aggregate, our data demonstrate the differential effect of MyD88 deficiency in donor T cells on GVL activity and GVHD severity in the allo-SCT setting.

\section{Absence of MyD88 in donor T cells leads to an increase of} donor CD4 and CD8 ${ }^{+} \mathrm{T}$ effector/memory cells in lymphoid organs of GVHD hosts

To elucidate host-specific responses in donor T cells in accordance with MyD88 signaling, the in vivo proliferation of donor $\mathrm{T}$ cells in the spleen of the two different GVHD hosts via CFSE staining of T cells was examined on day 4 post-allo-SCT. The proportion of low CFSE incorporation by $\mathrm{CD}^{+}$and $\mathrm{CD}^{+} \mathrm{T}$ cells was not significantly different between the two groups (Fig. 3A).

Next, we determined donor T-cell activation after allo-SCT according to the presence of MyD88 signaling. To more clearly compare the extent of tissue infiltration by donor $\mathrm{T}$ cells between the two groups of GVHD hosts, lethally irradiated F1 $(\mathrm{H}-$ $2^{\mathrm{b} / \mathrm{d}}$ ) mice were transplanted with TCD-BM cells isolated from congenic B6.Ly-5a B6 $\left(\mathrm{H}-2^{b+} \mathrm{CD} 45.1^{+}\right)$mice and T cells from B6 $\left(\mathrm{H}-2^{b+} \mathrm{CD} 45.1^{-}\right)$mice. In the peripheral blood of allogeneic recipients from day 7 to day 28 after allo-SCT, the proportions of donor T-cell derived $\mathrm{CD}^{+}$and $\mathrm{CD}^{+} \mathrm{T}$ cells and their effector/memory phenotype were comparable between the two groups (Fig. 3B). To investigate the contribution of MyD88 deficiency in the donor T-cell component to the development of the T effector cell subsets in the allogeneic recipients, we examined the proportion of $\mathrm{CD}^{+}$and $\mathrm{CD} 8^{+} \mathrm{T}$ cells within the spleen and peripheral lymph nodes (PLN), wherein donor T cells are activated by host antigen presenting cells to elucidate host-specific responses in donor T cells at day 28 after allo-SCT. As shown in Fig. 3C, on day 28 absolute cell number within the spleen and PLN was increased in recipient mice receiving B6.MyD88KO T cells as compared with mice receiving B6.WT $\mathrm{T}$ cells. In preliminary experiments using B6.Ly-5a mice as donors, we determined by FACS analysis that all (> 99\%) CD4 ${ }^{+}$ and $\mathrm{CD}^{+}$cells were of donor origin by days 28 in both the spleen and skin tissues (data not shown). The frequencies of $\mathrm{H}$ $2^{\mathrm{d}-} \mathrm{CD} 45.1^{-} \mathrm{T}$ cells in spleen and $\mathrm{CD} 4^{+} \mathrm{H}-2^{\mathrm{d}-} \mathrm{CD} 45.1^{-} \mathrm{T}$ cells in PLN that originated from the donor T-cell component were lower in the recipients of MyD88KO T cells than in those of WT T 
A

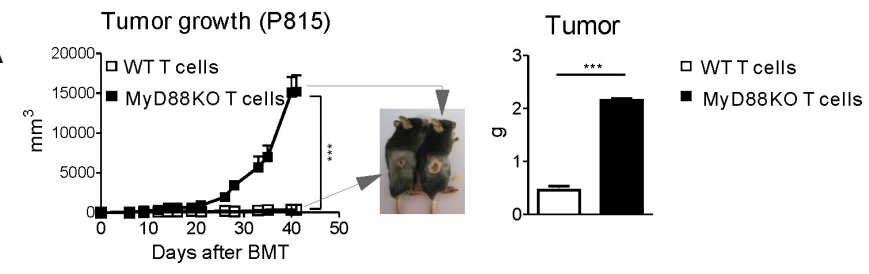

Tumor growth (L1210)

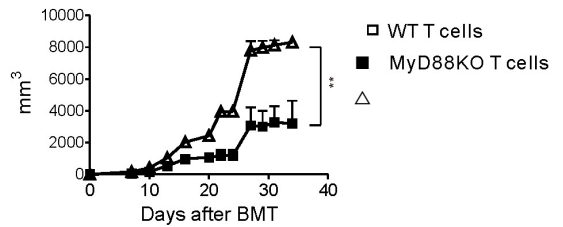

B
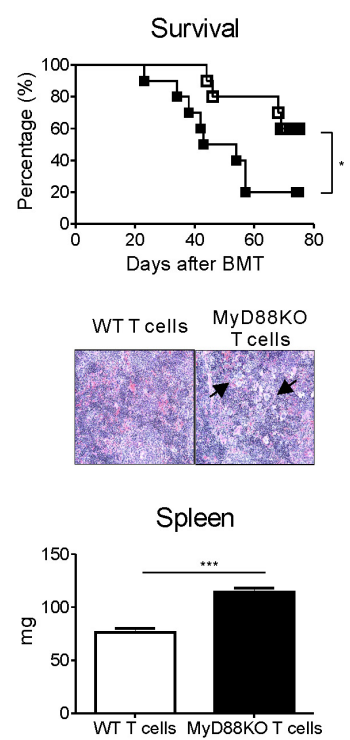

口WT T cells

- MyD88KO T cells

C
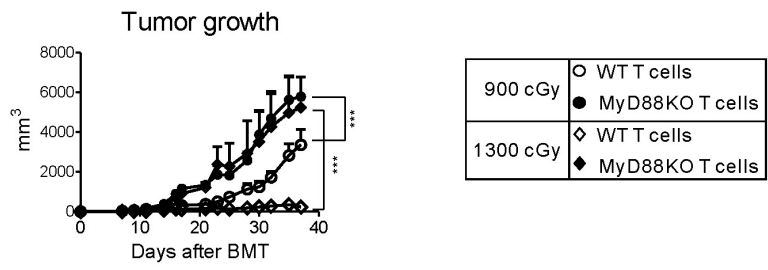

D
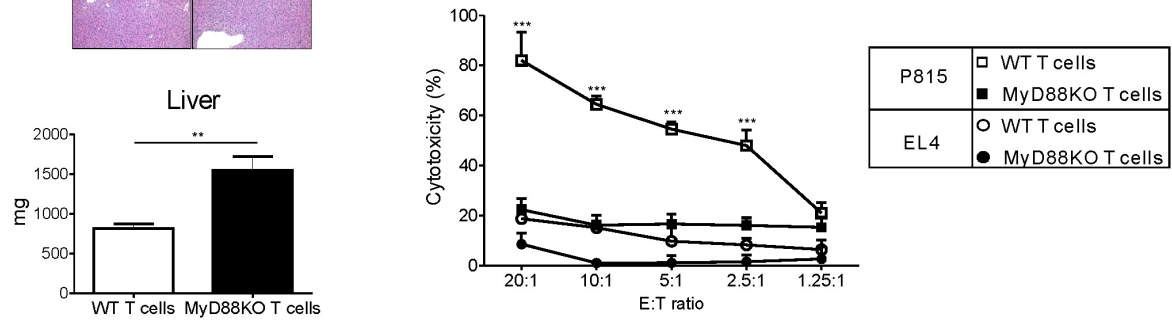

E

IFN- $\gamma$

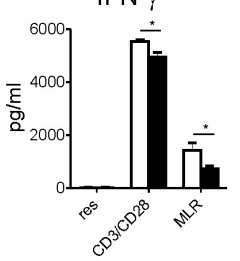

IL-6

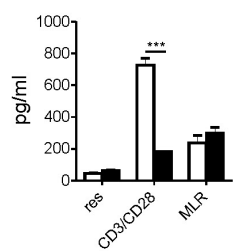

granzyme B

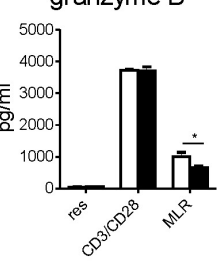

IL-17

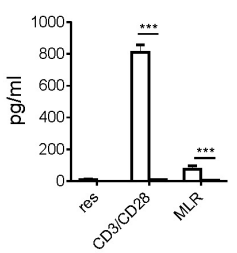

IL-10

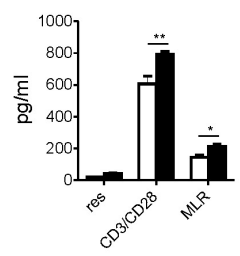

TNF- $\alpha$

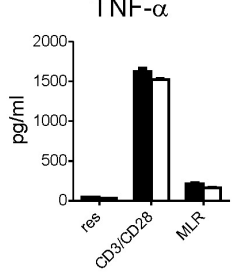

aWT T cells

- MyD88KO T cells

Fig. 1. Deficiency of MyD88 in donor $T$ cells mitigates GVL effect. Lethally irradiated recipient mice were transplanted with WT TCD-BM $(5 \times$ $10^{6}$ ) together with either WT or MyD88KO mice spleen T cells $\left(1 \times 10^{6}\right)$ on day 0 . (A) Recipient mice were injected s.c with $1 \times 10^{6}$ P815 cells (left) or L1210 cells (right) on day 1 post-transplantation. Tumor growth was monitored every 3 or 4 days after s.c tumor inoculation. Tumor measurement was finally determined on day 42 . Mice were sacrificed and tumor weights were measured $\left({ }^{* * *} P<0.001\right)$. Data are presented as mean \pm SEMs and are representative of duplicate experiment $(n=12$ per groups). (B) Lethally irradiated recipient mice were transplanted with WT TCD-BM $\left(5 \times 10^{6}\right)$ together with either WT or MyD88KO mice spleen T cells $\left(1 \times 10^{6}\right)$ on day 0 . Thereafter recipient mice were injected i.v with $3 \times 10^{3}$ P815 tumor cells on day 1 . Kaplan-Meier survival curve of the transplanted mice (top, $n=10$ per group). Percent survival after transplantation, WT T cells vs. MyD88KO T cells by the wilcoxon rank-sum test ( $\left.{ }^{*} \mathrm{P}<0.05\right)$. Mice were sacrificed and spleen and liver weights were measured on day 48 (bottom, $n=5$ per group; ${ }^{* *} P<0.01$ or ${ }^{* * *} P<0.001$ ). Arrows indicate the aggregation of leukemic cells. Data are presented as means \pm SEMs and representative of duplicate experiment $(n=10$ per groups). (C) Recipient mice were irradiated with 900 ( $\circ$ and $\bullet, n=6$ ) or $1300 \mathrm{cGy}\left(\diamond\right.$ and $\diamond, \mathrm{n}=6$ ) TBI and injected with $5 \times 10^{6}$ TCD BM together with either WT or MyD88KO mice spleen T cells $\left(1 \times 10^{6}\right)$ on day 0 . All animals were also injected s.c. with $1 \times 10^{6} \mathrm{P} 815$ tumor cells on day 1 and tumor growth was monitored $\left({ }^{\star * *} \mathrm{P}<\right.$ 0.001). Data from one of two replicate experiments that yielded similar results are shown. Data are presented as means $\pm S E M s$. (D, F) B6 WT or MyD88KO CD3 ${ }^{+}$T cells were co-cultured with F1 stimulators in a primary mixed lymphocyte reaction (MLR). (D) After 4 days of MLR, purified CD8 ${ }^{+}$cells were assessed for cytotoxicity against P815 $\left(\mathrm{H}-2^{\mathrm{d}}\right.$, $\square$ and $\left.\mathbf{m}, \mathrm{n}=4\right)$ or EL4 $\left(\mathrm{H}-2^{\mathrm{b}}\right.$, $\circ$ and $\left.\bullet, n=4\right)$ target cells. $(F)$ Culture supernatants from MLR were collected and assayed for IFN- $\gamma$, IL-6, granzyme B, IL-17, IL-10 and TNF- $\alpha$ production. Data shown (D, F) are representative of two independent experiments. Data are presented as means $\pm S E M s$. ${ }^{*} P<0.05,{ }^{* *} P<0.01$ or ${ }^{* *} P<0.001$. 
$\boldsymbol{A}$
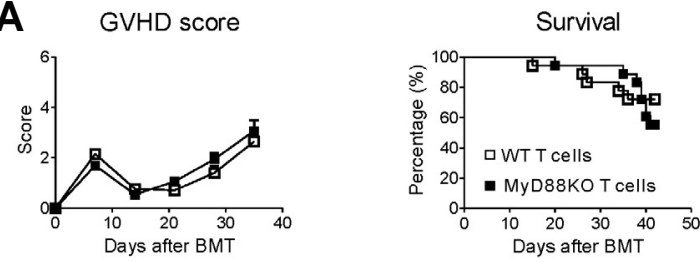

B

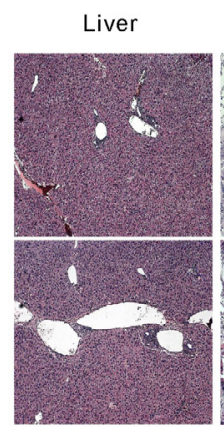

Lung
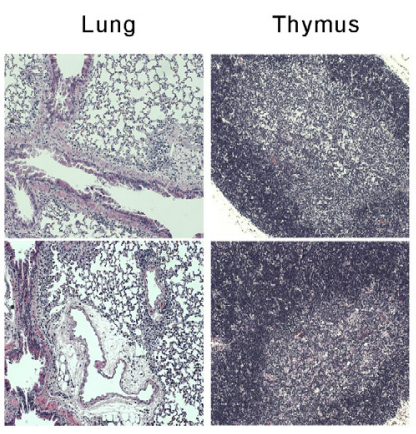

Small Intestine

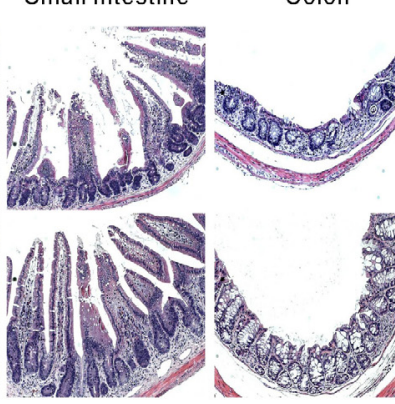

WT T cells
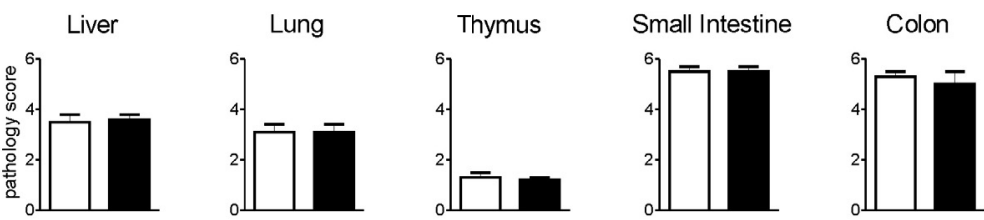

$\square$ WT T cells

- MyD88KO T cells

C

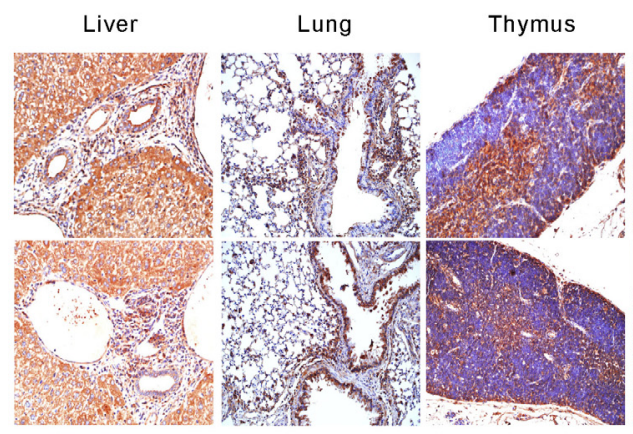

Colon

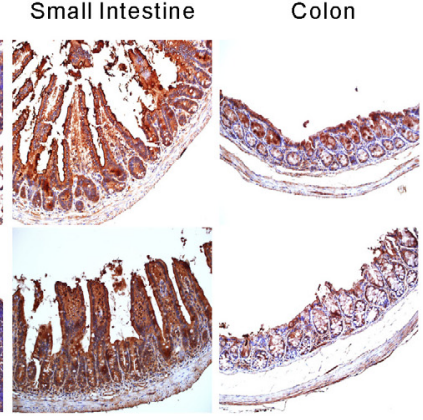

WT T cells

MyD88KO T cells

MyD88KO T cells

Fig. 2. Deficiency of MyD88 in donor T cells does not attenuate GVHD severity. Lethally irradiated recipient mice were transplanted with WT TCD-BM $\left(5 \times 10^{6}\right)$ together with either WT or MyD88KO mice spleen $T$ cells $(1 \times$ $10^{6}$ ) on day 0 . Animals were assessed weekly with respect to the clinical severity of aGVHD. (A) Corresponding GVHD scores (left) and KaplanMeier survival curve (right) of the transplanted mice. Data are presented as mean \pm SEMs and are representative of duplicate experiment $(\mathrm{n}=10$ per groups). (B) Representative histopathologic examination of H\&E stained sections of paraffinembedded liver, lung, thymus, small intestine and colon. Pathology scores are presented as means \pm SEMs. (C) Representative expressions of TNF- $\alpha$ in each organ are shown. cells. However, the absolute numbers of the donor $\mathrm{H}-2^{\mathrm{d}-}$ CD45.1 $\mathrm{T}$ cells were significantly higher in each organ of the former than in the latter because the total cellularity levels were higher in the former. Similar phenomena were observed in the effector/memory cell components $\left(\mathrm{CD} 44^{\text {high }} \mathrm{CD} 62 \mathrm{~L}^{\text {low }}\right)$ of the donor $\mathrm{H}-2^{\mathrm{d}-} \mathrm{CD} 45.1^{-} \mathrm{T}$ cells. These data indicate that the transferred donor $T$ cells could more actively differentiate into effector-memory $\mathrm{T}$ cells even in the absence of the MyD88dependent pathway within donor T cells.

We also evaluated the potential mechanisms for inflammation-mediated suppression of GVL effect with preservation of aGVHD severity mediated by donor T cells. Previous studies in murine allo-SCT models have demonstrated the important role for IFN- $\gamma$ and TNF- $\alpha$ in the development of both GVHD and the GVL effect (Schmaltz et al., 2003; Yang et al., 2002). To ask whether the diminished GVL effect with preservation of aGVHD severity after transplantation with donor T cells of MyD88KO donor was due to skewed production of inflammatory cytokines, we measured the levels of these cytokines in recipient sera on days 7 and 28 after transplantation. We found a significant decrease in serum concentrations of IFN- $\gamma$, IL-6, TNF- $\alpha$ and IL17A on day 7 in the recipients of MyD88KO T cells compared with those of WT T cells. In contrast, circulating levels of TNF- $\alpha$, MCP-1 and RANTES on day 28 were significantly increased in the former compared with the latter (Fig. 3D). Altogether, these data indicated that MyD88KO T cells transplanted into allogeneic recipients retained their capacity to proliferate against allogeneic stimuli and equally contributed to the occurrence of aGVHD compared with WT T cells, while not mediating the GVL effect with skewed cytokine productions after allo-SCT.

Absence of MyD88 in donor T cells leads to an expansion of Treg and TH2 cells with a decrease of TH1 cells in lymphoid organs

We next addressed whether the absence of MyD88 in donor T cells affected the generation of $\mathrm{TH} 1, \mathrm{TH} 2$, Treg and $\mathrm{TH} 17$ cells 
A

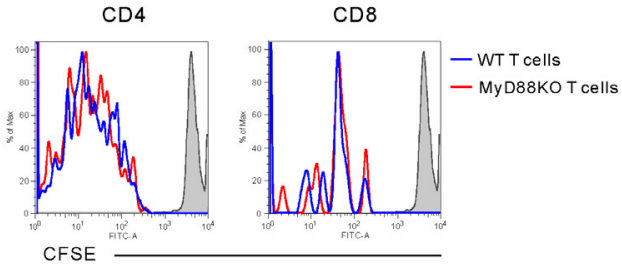

B
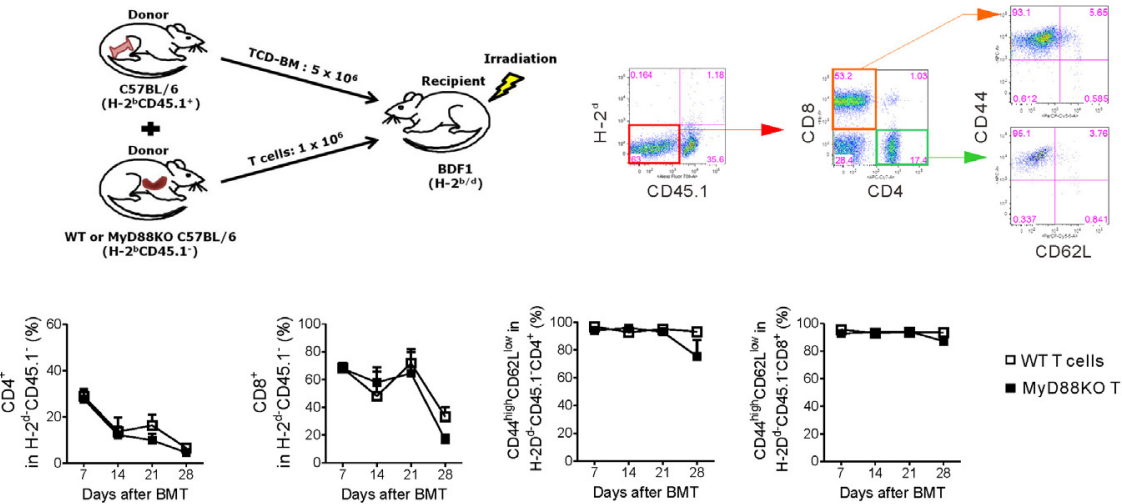

C
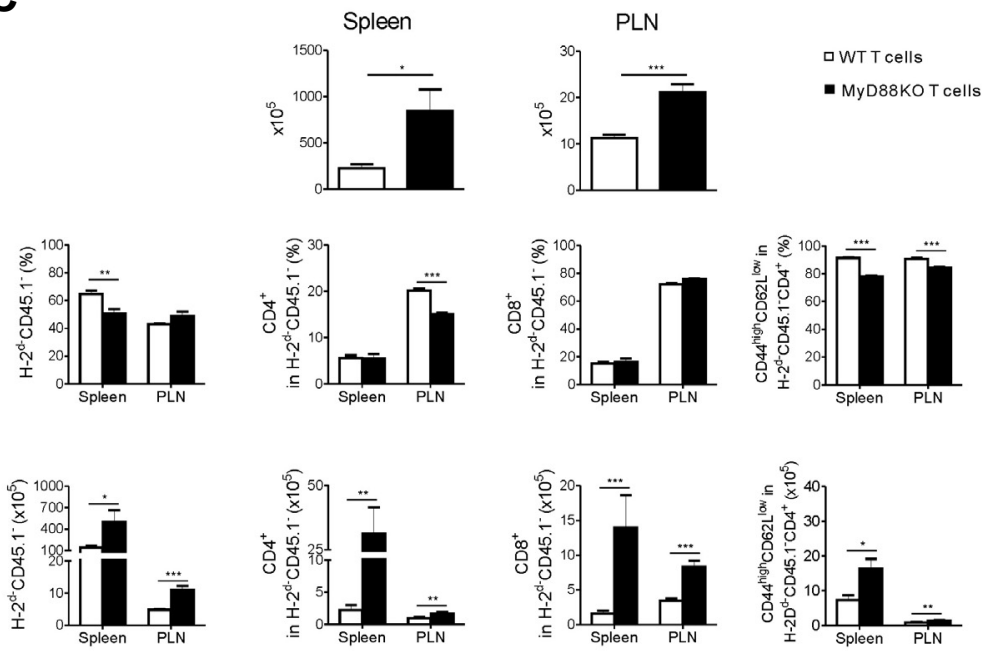

D
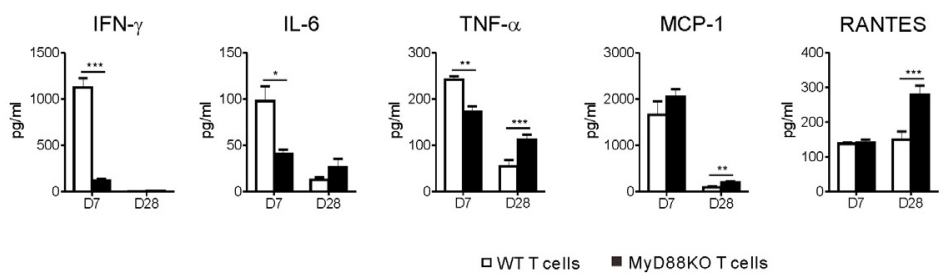

$\square$ WT T cells

- MyD88Ko T cells
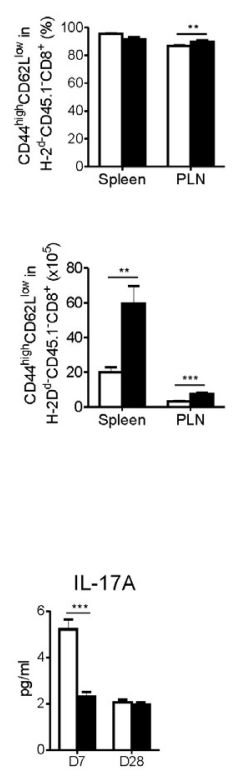

Fig. 3. Absence of MyD88 in donor $T$ cells leads to an increase of donor CD4 and CD8 T effector/memory cells in lymphoid organs of GVHD hosts. (A) Lethally irradiated recipient mice were given $5 \times 10^{6}$ WT TCD-BM cells $\left(\mathrm{CD} 45.1^{+}\right)$plus $1 \times 10^{6}$ purified WT or MyD88KO mice spleen T cells and in vivo proliferation was determined on day 4 . Recipient mice received CFSElabeled WT or MyD88KO T cells, as described in "Materials and Methods" ( $\mathrm{n}=5$ per groups; ${ }^{*} \mathrm{P}<0.05$ or $\left.{ }^{* *} \mathrm{P}<0.01\right)$. Data from one of two independent experiments are shown. Data are presented as means \pm SEMs. (B-D) Lethally irradiated recipient mice were given $5 \times$ $10^{6}$ WT TCD-BM cells (CD45. $\left.1^{+}\right)$ plus $1 \times 10^{6}$ purified WT or MyD88KO mice spleen $T$ cells (CD45.1'). (B) Representative FACS analysis of donor T-cell derived $\left(\mathrm{CD} 45.1^{-} \mathrm{H}-2 \mathrm{~d}^{-}\right) \mathrm{CD}^{+}$and $\mathrm{CD}^{+}$and their effector/memory T cells (CD45.1 ${ }^{-1} \mathrm{H}-2 \mathrm{~d}^{-} \mathrm{CD} 4^{+} \mathrm{CD} 62$ $\mathrm{L}^{\text {low }} \mathrm{CD} 44^{\text {hi }}$ or $\mathrm{CD} 45 . \mathrm{H}^{-} 2 \mathrm{~d}^{-} \mathrm{CD} 8^{+}$ $\left.\mathrm{CD}^{\mathrm{CL}} \mathrm{L}^{\mathrm{low}} \mathrm{CD} 44^{\mathrm{hi}}\right)$ were examined on blood cells ( $n=6$, each group). Data are presented as means \pm SEMs representative of duplicate experiments. (C) The expansion of donor Tcell derived $\mathrm{CD}^{+}$and $\mathrm{CD}^{+}{ }^{+} \mathrm{T}$ cells and their effector/memory $T$ cells in the spleen and peripheral lymph node (PLN) were assessed on day 28 posttransplantation $(n=6$, each group). Data are presented as means \pm SEMs and are representative of duplicate experiments. (D) Serum samples from recipient mice ( $\mathrm{n}=6$, each group) that underwent transplantation were obtained on days 7 and 28 post-transplantation. Serum IFN- $\gamma$, IL-6, TNF- $\alpha$, MCP1 , RANTES and IL-17A levels were determined by Cytometric bead analysis. Data from one of two independent experiments are shown. Data are presented as means \pm SEMs. Statistics: *P $<0.05,{ }^{* *} \mathrm{P}<0.01$ or ${ }^{* * *} \mathrm{P}<$ 0.001 .

while the frequencies of $\mathrm{CD}^{+}$and $\mathrm{CDB}^{+} \mathrm{T}$ cells producing IL-4 and Foxp3 from spleen were significantly increased. On day 28, however, the expressions of IL-4 and Foxp3 in both splenic $T$ cell subsets were significantly decreased in the 
CD4 T cells

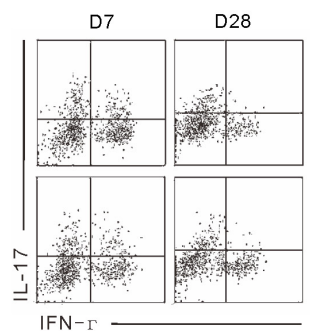

D7

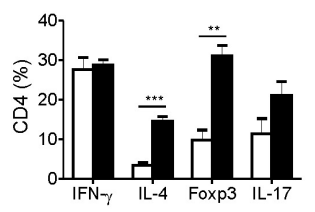

$B$

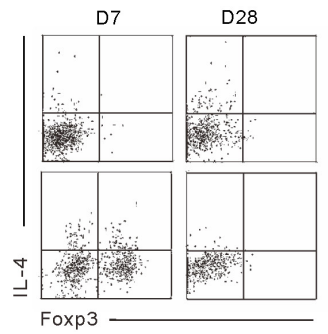

D28

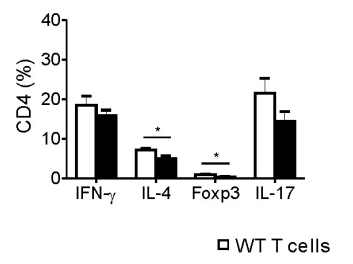

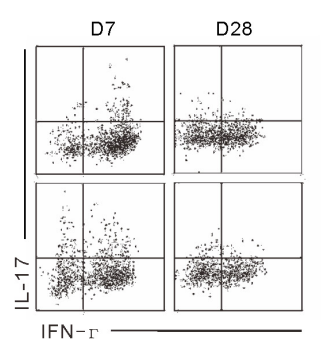

D7

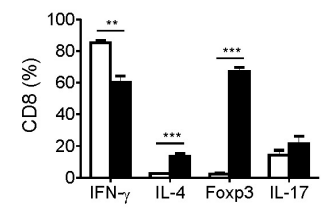

- MyD88KO T cells

CD8 T cells

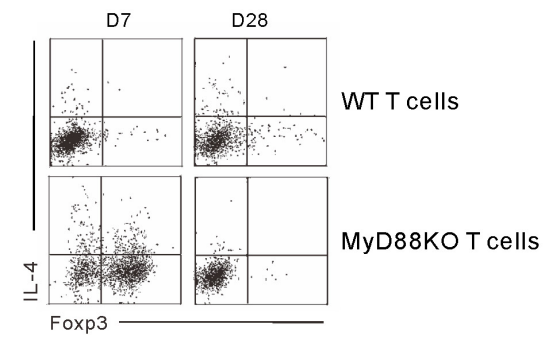

D28

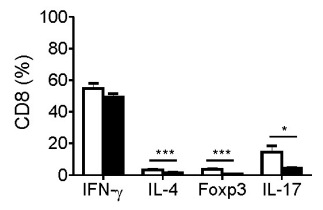

TDLN

D7

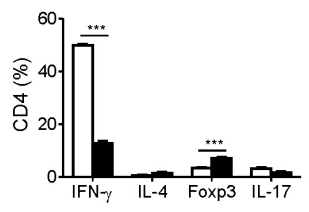

D28

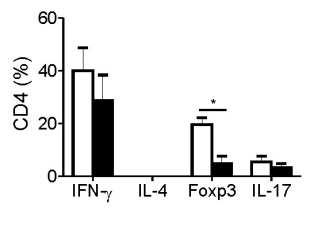

D7

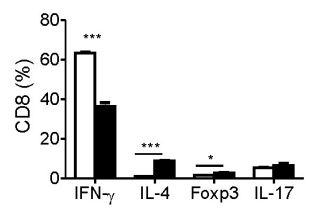

MyD88Ko T cells
D28

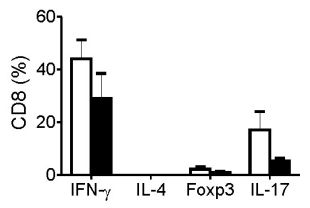

C

Spleen

D7

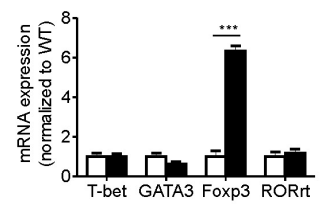

D28

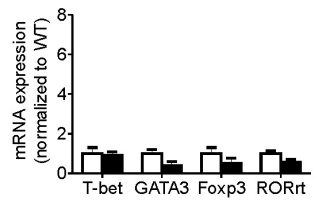

WWT T cells

TDLN

D7

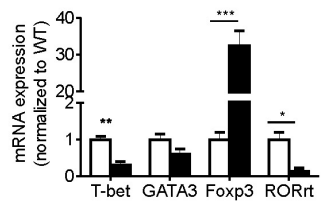

D28

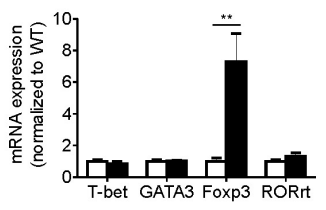

Fig. 4. Absence of MyD88 in donor T cells leads to an expansion of Treg and TH2 cells with reduced TH1 cells in lymphoid organs. Lethally irradiated recipient mice were transplanted with WT TCD-BM $\left(5 \times 10^{6}\right)$ together with either WT or MyD88KO mice spleen T cells $\left(1 \times 10^{6}\right)$ on day 0 . All animals $\left(n=6\right.$, each group) were also injected s.c. with $1 \times 10^{6}$ P815 tumor cells on day 1 . Spleens (A) and tumor-draining lymph node (TDLN) (B) were harvested from the recipient mice on days 7 and 28 post-transplantation. Expressions of IFN- $\gamma$, IL-4, Foxp3 and IL-17 on the CD4 ${ }^{+}$and $C D 8^{+}$T cells were assayed by FACS. (C) Expression of T-bet, GATA3, Foxp3 and ROR 7 were measured in the mononuclear cells of spleen and TDLN using quantitative RT-PCR. Data are presented as means \pm SEMs and are representative of duplicate experiments $\left({ }^{*} P<0.05,{ }^{* *} P<0.01\right.$ or $\left.{ }^{* \star *} P<0.001\right)$.

former as compared with the latter. We also analyzed the secretion of IFN- $\gamma$, IL-4 and Foxp3 by $\mathrm{CD} 4^{+}$and $\mathrm{CD} 8^{+} \mathrm{T}$ cells isolated from tumor-draining lymph nodes (TDLN). Similarly, the capacity of those cells to secrete IFN- $\gamma 7$ days after transplantation was significantly diminished in MyD88KO T-cell recipients as compared with WT T-cell recipients, while the fre- quencies of $\mathrm{CD}^{+}$and $\mathrm{CD}^{+} \mathrm{T}$ cells expressing IL-4 and Foxp3 were significantly increased (Fig. 4B). $\mathrm{CD}^{+}{ }^{+} \mathrm{Foxp}^{+}$cells were also decreased in the former as compared with the latter on day 28. Regarding TH17 cells, we could not observe any differences in the proportion of IL-17 secreting T cells at day 7 in spleen and TDLN from MyD88KO T-cell recipients as com- 
pared with WT T-cell recipients.

As MyD88 deficiency in donor T cells resulted in reduced GVL effect, we further analyzed whether inhibition of the GVL effect involved a Treg-dependent mechanism. On day 7 after allo-SCT, we also found a significant increase in the expression of Foxp3 among mononuclear cells from the spleen and TDLN (Fig. $4 \mathrm{C}$ ), suggesting that the increase in Treg cells, in particular, could contribute to the regulation of the alloreactive response against GVL effect. Accordingly, on day 28 Foxp3 expression in TDLN remained elevated on day 28 .

\section{DISCUSSION}

Thus far, the role of MyD88 within T cells has not been extensively addressed in the context of the aGVHD and GVL effect after allo-SCT. Our data show that MyD88 in the donor T cells contributes to the maintenance of GVL effect regardless of the severity of aGVHD. Donor $T$ cell expansion with effector/memory T-cell differentiation were more greatly enhanced in GVHD hosts of MyD88KO T cells. Recipients of MyD88KO T cells had a markedly increased expansion of Foxp3- and IL4expressing $T$ cells with reduced INF- $\gamma$ producing $T$ cells in the spleen and TDLN early after transplantation compared with recipients of WT T cells. In vitro, absence of MyD88 in T cells results in defective cytolytic activity to tumor targets with less ability to produce IFN- $\gamma$ or granzyme B that are known as critical for GVL effect. MyD88 signaling by donor T cell may be required for proper GVL effect but not for development of aGVHD and these results provide a link between an innate defect and the anti-leukemic effect of donor T cells in the setting of allo-SCT.

Given the limitations of existing immunosuppressive therapies, a better understanding of the molecular mechanisms that govern GVHD versus GVL effect are urgently needed. Currently, in the pathogenesis of aGVHD, a widely accepted model postulates that intensive chemotherapy and/or TBI during pretransplant conditioning results in tissue damage and a loss of epithelial barrier function (Hill and Ferrara, 2000). Subsequent translocation of bacterial components as well as release of endogenous danger molecules stimulate pattern-recognition receptors of host antigen-presenting cells to trigger the production of pro-inflammatory cytokines (cytokine storm) that modulate $T$ cell allo-reactivity against host tissues, but eventually also the beneficial GVL effect. After allo-SCT donor T cells undergo an initial phase of expansion and differentiation into functional effectors. Thus, developing a better understanding of the signals that regulate the generation, maintenance, and reactivation of donor-derived memory $T$ cells is central to successful allo-SCT. During the development of aGVHD, activated effector $\mathrm{T}$ cells exit lymphoid tissues and traffic to parenchymal target organs, such as, intestine, liver, skin, and lungs where they cause tissue damage (Wysocki et al., 2005). In this study, the absence of the MyD88 in donor T cells has been found to increase expansion of alloreactive effector/memory $T$ cells in spleen and PLN, contributing to an integral role in GVHD and GVL effect. A similar degree of tissue destruction in aGVHD target organs, however, was observed in both groups, suggesting that the expanded allo-reactive $T$ cells, even if absent MyD88, persist as a stable memory population that differs both phenotypically and functionally from the naive T-cell population and some portion of these memory $T$ cells could differentiate into cytotoxic $T$ cells with long-lasting allo-reactivity, contributing to the perpetuation of damage of the GVHD organs.

GVL effect depends on the presence of tumor antigen-primed $\mathrm{CD}^{+}$or $\mathrm{CD}^{+}$effector $\mathrm{T}$ cells, the ability of such $\mathrm{T}$ cells to traffic to sites of leukemic growth, the persistence of cells in sufficient concentrations at tumor sites, and the capacity of $\mathrm{T}$ cells to maintain cytotoxic effector functions in the face of local immunosuppressive mechanisms (Dudley et al., 2005). We have previously showed that a higher TBI dose (1300 vs. 900 cGy) resulted in a significant reinforcement of the GVL effect, and mechanistically, this is likely to be caused by alterations in effector T cell trafficking into the tumor tissue (Lim et al., 2011). In the present study, increased aGVHD severity by intensifying conditioning, however, did not translate into enhanced GVL effect when MyD88 is deficient in donor T cells, suggesting that the GVHD-induced GVL effects were not observed in the allogeneic recipients of MyD88KO T cells and maintaining MyD88expressing effector $\mathrm{T}$ cells within the tumor microenvironment are viewed favorably in terms of reducing leukemia relapse after allo-SCT.

We also observed a significant increase of $\mathrm{CD}^{+}$or $\mathrm{CD}^{+}{ }^{+}$oxp $^{+}$Treg cells as well as Foxp3 expression in the spleen and TDLN within the recipients administered with MyD88KO $T$ cells although the mechanism through which MyD88 deficiency results in Treg expansion is not clear. The protective effect of Treg on aGVHD has already been demonstrated in several models (Shin et al., 2011; Taylor et al., 2002). In the recipients of MyD88KO T cells enhancing Tregs and TH2 cells and decreasing TH1 cells in spleen and TDLN early after transplantation but decreasing Tregs and TH2 after the lapse of time may be associated with reducing GVL effect rather than the GVHD-associated tissue damage. We assume that the kinetics of pro-inflammatory cytokine production would be in line with the dissociation between GVHD and GVL effect (Fig. 3D). The contribution of $\mathrm{TH} 17$ cells in aGVHD is somewhat controversial. In our study, we did not detect any differences in IL-17A production by T cells 1 week after allo-SCT but serum levels of IL-17A were significantly reduced in the recipients of MyD88KO T cells. Interestingly, production of IL-17A from MyD88 KO T cells was markedly reduced in $\mathrm{T}$ cells from MyD88KO compared to those from WT mice (Chang et al., 2013).

In this donor/recipient strain combination (B6 $\rightarrow$ B6D2F1), GVHD mortality is critically dependent on donor $\mathrm{CD} 4^{+} \mathrm{T}$ cells, while $\mathrm{CD}^{+} \mathrm{T}$ cells that mediate cytotoxicity are more potent effectors of GVL effect (Schmaltz et al., 2001; Teshima et al., 1999; Yang et al., 1997). Taking into account the comparable severity of aGVHD, the host-specific responses in MyD88KO donor $\mathrm{CD}^{+} \mathrm{T}$ cells might be preserved. In vitro, however, the absence of MyD88 results in defective cytolytic activity in CD8 ${ }^{+}$ $\mathrm{T}$ cells to tumor and a reduced ability to secrete proinflammatory cytokines to host antigens. Subsequently, functional dissociation of the two T-cell subsets according to MyD88 deficiency in T cells might be a cause of maintaining GVHD severity with reduced GVL effect.

In summary, these data demonstrate that the MyD88 of donor $T$ cells is critical for preservation of GVL activity regardless of the occurrence of aGVHD in this allo-SCT model, offering the opportunity for improving GVL activity by targeting TLR-MyD88 signaling within donor T cells. The dissociation of GVL effect from GVHD may ultimately allow us to design new effective therapeutic modality against hematologic malignancies, which can harness the beneficial GVL effect and reduce the toxicity of GVHD.

\section{ACKNOWLEDGMENTS}

This study was supported by the Korea Healthcare Technology R\&D Project, Ministry of Health, Welfare, and Family Affairs, Republic of Korea (Grant no. A120262). 


\section{REFERENCES}

Appelbaum, F.R. (2001). Haematopoietic cell transplantation as immunotherapy. Nature 411, 385-389.

Asprodites, N., Zheng, L., Geng, D., Velasco-Gonzalez, C., Sanchez-Perez, L., and Davila, E. (2008). Engagement of Tolllike receptor-2 on cytotoxic T-lymphocytes occurs in vivo and augments antitumor activity. FASEB J. 22, 3628-3637.

Barrett, A.J. (1997). Mechanisms of the graft-versus-leukemia reaction. Stem Cells 15, 248-258.

Bendigs, S., Salzer, U., Lipford, G.B., Wagner, H., and Heeg, K. (1999). CpG-oligodeoxynucleotides co-stimulate primary T cells in the absence of antigen-presenting cells. Eur. J. Immunol. 29, 1209-1218.

Caron, G., Duluc, D., Frémaux, I., Jeannin, P., David, C., Gascan, H., and Delneste, Y. (2005). Direct stimulation of human T cells via TLR5 and TLR7/8: flagellin and R-848 up-regulate proliferation and IFN-gamma production by memory $\mathrm{CD} 4+\mathrm{T}$ cells. J. Immunol. 175, 1551-1557.

Chang, J., Burkett, P.R., Borges, C.M., Kuchroo, V.K., Turka, L.A., and Chang, C.H. (2013). MyD88 is essential to sustain mTOR activation necessary to promote $T$ helper 17 cell proliferation by linking IL-1 and IL-23 signaling. Proc. Natl. Acad. Sci. USA 110, 2270-2275

Cooke, K. R., Hill, G. R., Crawford, J. M., Bungard, D., Brinson, Y. S., Delmonte, J., Jr., and Ferrara, J.L. (1998). Tumor necrosis factoralpha production to lipopolysaccharide stimulation by donor cells predicts the severity of experimental acute graft-versus-host disease. J. Clin. Invest. 102, 1882-1891.

Cottalorda, A., Verschelde, C., Marçais, A., Tomkowiak, M., Musette, P., Uematsu, S., Akira, S., Marvel, J., and Bonnefoy-Berard, N. (2006). TLR2 engagement on CD8 T cells lowers the threshold for optimal antigen-induced T cell activation. Eur. J. Immunol. 36, 1684-1693.

Dudley, M.E., Wunderlich, J.R., Yang, J.C., Sherry, R.M., Topalian, S.L., Restifo, N.P., Royal, R.E., Kammula, U., White, D.E., Mavroukakis, S.A., et al. (2005). Adoptive cell transfer therapy following non-myeloablative but lymphodepleting chemotherapy for the treatment of patients with refractory metastatic melanoma. J. Clin. Oncol. 23, 2346-2357.

Fukata, M., Breglio, K., Chen, A., Vamadevan, A.S., Goo, T., Hsu, D., Conduah, D., Xu, R., and Abreu, M.T. (2008). The myeloid differentiation factor 88 (MyD88) is required for CD4+ T cell effector function in a murine model of inflammatory bowel disease. J. Immunol. 180, 1886-1894.

Gelman, A.E., Zhang, J., Choi, Y., and Turka, L.A. (2004). Toll-like receptor ligands directly promote activated $C D 4+T$ cell survival. J. Immunol. 172, 6065-6073.

Hartman, Z.C., Osada, T., Glass, O., Yang, X.Y., Lei, G.J., Lyerly, H.K., and Clay, T.M. (2010). Ligand-independent toll-like receptor signals generated by ectopic overexpression of MyD88 generate local and systemic antitumor immunity. Cancer Res. 70, 72097220.

Hill, G.R., and Ferrara, J.L. (2000). The primacy of the gastrointestinal tract as a target organ of acute graft-versus-host disease: rationale for the use of cytokine shields in allogeneic bone marrow transplantation. Blood 95, 2754-2759.

Horowitz, M.M., Gale, R.P., Sondel, P.M., Goldman, J.M., Kersey, J., Kolb, H.J., Rimm, A.A., Ringden, O., Rozman, C., Speck, B., et al. (1990). Graft-versus-leukemia reactions after bone marrow transplantation. Blood 75, 555-562.

Kawai, T., Adachi, O., Ogawa, T., Takeda, K., and Akira, S. (1999). Unresponsiveness of MyD88-deficient mice to endotoxin. Immunity 11, 115-122.

Komai-Koma, M., Jones, L., Ogg, G.S., Xu, D., Liew, F.Y. (2004). TLR2 is expressed on activated T cells as a costimulatory receptor. Proc. Natl. Acad. Sci. USA 101, 3029-3034.
Lim, J.Y., Choi, M.S., Youn, H., Choi, E.Y., and Min, C.K. (2011). The influence of pretransplantation conditioning on graft-vs.leukemia effect in mice. Exp. Hematol. 39, 1018-1029.

Lim, J.Y., Cho, B.S., Min, C.K., Park, G., Kim, Y.J., Chung, N.G., Jeong, D.C., and Min, W.S. (2014). Fluctuations in pathogenic CD4+ T-cell subsets in a murine sclerodermatous model of chronic graft-versus-host disease. Immunol. Invest. 43, 41-53.

Min, C.K., Maeda, Y., Lowler, K., Liu, C., Clouthier, S., Lofthus, D. Weisiger, E., Ferrara, J.L., and Reddy, P. (2004). Paradoxical effects of interleukin-18 on the severity of acute graft-versus-host disease mediated by CD4+ and CD8+ T-cell subsets after experimental allogeneic bone marrow transplantation. Blood 104, 3393-3399.

Schmaltz, C., Alpdogan, O., Horndasch, K.J., Muriglan, S.J., Kappel, B.J., Teshima, T., Ferrara, J.L., Burakoff, S.J., and van den Brink, M.R. (2001). Differential use of Fas ligand and perforin cytotoxic pathways by donor T cells in graft-versus-host disease and graftversus-leukemia effect. Blood 97, 2886-2895.

Schmaltz, C., Alpdogan, O., Muriglan, S.J., Kappel, B.J., Rotolo, J.A., Ricchetti, E.T., Greenberg, A.S., Willis, L.M., Murphy, G.F., Crawford, J.M., et al. (2003). Donor T cell-derived TNF is required for graft-versus-host disease and graft-versus-tumor activity after bone marrow transplantation. Blood 101, 2440-2445.

Schnare, M., Barton, G.M., Holt, A.C., Takeda, K., Akira, S., and Medzhitov, R. (2001). Toll-like receptors control activation of adaptive immune responses. Nat. Immunol. 2, 947-950.

Shin, H.J., Baker, J., Leveson-Gower, D.B., Smith, A.T., Sega, E.I., and Negrin, R.S. (2011). Rapamycin and IL-2 reduce lethal acute graft-versus-host disease associated with increased expansion of donor type CD4+CD25+Foxp3+ regulatory T cells. Blood 118, 2342-2350.

Taylor, P.A., Lees, C.J., and Blazar, B.R. (2002). The infusion of ex vivo activated and expanded CD4(+)CD25(+) immune regulatory cells inhibits graft-versus-host disease lethality. Blood 99, 34933499.

Teshima, T., Hill, G.R., Pan, L., Brinson, Y.S., van den Brink, M.R., Cooke, K.R., and Ferrara, J.L. (1999). IL-11 separates graftversus-leukemia effects from graft-versus-host disease after bone marrow transplantation. J. Clin. Invest. 104, 317-325.

Tomita, T., Kanai, T., Fujii, T., Nemoto, Y., Okamoto, R., Tsuchiya, K., Totsuka, T., Sakamoto, N., Akira, S., and Watanabe, M. (2008). MyD88-dependent pathway in T cells directly modulates the expansion of colitogenic CD4+ T cells in chronic colitis. J. Immunol. 180, 5291-5299.

van den Brink, M.R., and Burakoff, S.J. (2002). Cytolytic pathways in haematopoietic stem-cell transplantation. Nat. Rev. Immunol. 2, 273-281.

Wu, H., Chen, G., Wyburn, K.R., Yin, J., Bertolino, P., Eris, J.M., Alexander, S.I., Sharland, A.F., and Chadban, S.J. (2007). TLR4 activation mediates kidney ischemia/reperfusion injury. J. Clin. Invest. 117, 2847-2859.

Wysocki, C. A., Panoskaltsis-Mortari, A., Blazar, B. R., and Serody, J. S. (2005). Leukocyte migration and graft-versus-host disease. Blood 105, 4191-4199.

Yang, Y.G., Sergio, J.J., Pearson, D.A., Szot, G.L., Shimizu, A., and Sykes, M. (1997). Interleukin-12 preserves the graft-versusleukemia effect of allogeneic CD8 T cells while inhibiting CD4dependent graft-versus-host disease in mice. Blood 90, 46514660.

Yang, Y.G., Qi, J., Wang, M.G., and Sykes, M. (2002). Donorderived interferon gamma separates graft-versus-leukemia effects and graft-versus-host disease induced by donor CD8 T cells. Blood 99, 4207-4215.

Zheng, L., Asprodites, N., Keene, A.H., Rodriguez, P., Brown, K.D., and Davila, E. (2008). TLR9 engagement on CD4 T lymphocytes represses gamma-radiation-induced apoptosis through activation of checkpoint kinase response elements. Blood 111, 2704-2713. 